\title{
Medical Image Segmentation for Mobile Electronic Patient Charts Using Numerical Modeling of IoT
}

\author{
Seung-Hoon Chae, ${ }^{1}$ Daesung Moon, ${ }^{2}$ Deok Gyu Lee, ${ }^{3}$ and Sung Bum Pan ${ }^{4}$ \\ ${ }^{1}$ The Research Institute of IT, Chosun University, 309 Pilmun-daero, Dong-gu, Gwangju 501-759, Republic of Korea \\ ${ }^{2}$ Electronics and Telecommunications Research Institute, 161 Gajeong-dong, Yuseong-gu, Daejeon 305-350, Republic of Korea \\ ${ }^{3}$ Department of Information Security, Seowon University, 377-3 Musimseo-ro, Heungdeok-gu, Cheongju-si, \\ Choong-Chung Buk-do 361-742, Republic of Korea \\ ${ }^{4}$ Department of Electronics Engineering, Chosun University, 309 Pilmun-daero, Dong-gu, Gwangju 501-759, Republic of Korea
}

Correspondence should be addressed to Sung Bum Pan; sbpan@chosun.ac.kr

Received 16 December 2013; Accepted 31 March 2014; Published 12 June 2014

Academic Editor: Young-Sik Jeong

Copyright (C) 2014 Seung-Hoon Chae et al. This is an open access article distributed under the Creative Commons Attribution License, which permits unrestricted use, distribution, and reproduction in any medium, provided the original work is properly cited.

\begin{abstract}
Internet of Things (IoT) brings telemedicine a new chance. This enables the specialist to consult the patient's condition despite the fact that they are in different places. Medical image segmentation is needed for analysis, storage, and protection of medical image in telemedicine. Therefore, a variety of methods have been researched for fast and accurate medical image segmentation. Performing segmentation in various organs, the accurate judgment of the region is needed in medical image. However, the removal of region occurs by the lack of information to determine the region in a small region. In this paper, we researched how to reconstruct segmentation region in a small region in order to improve the segmentation results. We generated predicted segmentation of slices using volume data with linear equation and proposed improvement method for small regions using the predicted segmentation. In order to verify the performance of the proposed method, lung region by chest CT images was segmented. As a result of experiments, volume data segmentation accuracy rose from 0.978 to 0.981 and from 0.281 to 0.187 with a standard deviation improvement confirmed.
\end{abstract}

\section{Introduction}

Telemedicine is defined by the World Health Organization (WHO) as "the practice of medical care using interactive audiovisual and data communications. This includes the delivery of medical care services, diagnosis, consultation, treatment, as well as health education and the transfer of medical data" [1]. In 1906, Wilhelm Einthoven experimented the first telemedicine by transmitting electrocardiogram (ECG) recordings through telephone [2-4]. Since then, telemedicine has become routine practice for specialists to review remote patients' radiology and neurosurgery image $[5,6]$. If we use telemedicine, the information of patient's condition is checked using mobile device remotely as shown in Figure 1.

As a new generation information technology, Internet of Things (IoT) brings telemedicine a new chance, which applies sensors and network to traditional medical devices, and therefore is able to assign the intelligence to them and implement deeper communication and interaction between patients and remote specialists [7-10]. Besides patients' benefit, IoT even helps entire health industry, in which wide scope of medical devices are connected to existing health network, patient crucial life signal is captured by sensors and transmitted to remote medical center, and the doctor is able to remotely monitor patient condition and provide medical suggestion and aiding $[11,12]$.

By the improvement of the performance of medical imaging equipment, in accordance with the acquisition of high-resolution digital images, computer image analysis is being actively applied in the field of medical diagnosis and treatment. Recently, through various researches, computeraided diagnosis (CAD) system showed the results that can 

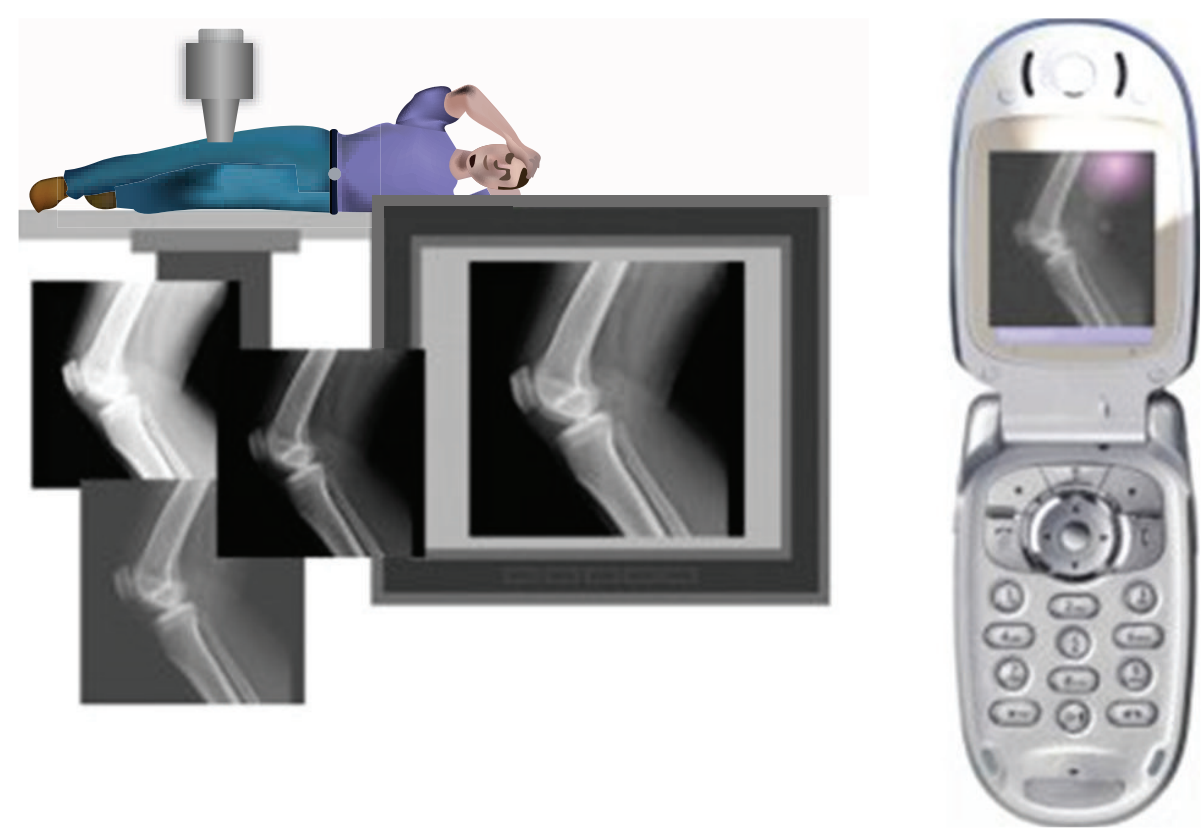

FIgURE 1: Example of telemedicine system.

enhance effect of diagnosis and treatment by assisting the specialists [13]. Especially, the field of medical imaging is growing rapidly by new ways to extract or visualize the organ tissue information from diagnostic medical images obtained by a variety of medical imaging equipment such as $\mathrm{X}$-ray, computerized tomography (CT), magnetic resonance imaging (MRI), ultrasound, and Positron emission tomography (PET) [14]. Ritter et al. divided the major issues in the field of medical image processing into image enhancement, image segmentation, image registration, quantification, visualization, and computer-aided detection [15]. Image segmentation of these is important image processing that needs to be ahead of a variety of medical image processing such as image registration, quantification, visualization, and computer-aided detection. Image segmentation is utilized for not only preprocessing stage of other images' processing but also image compression and protection of medical image. Medical images with high-resolution have difficulties in storage and transmission because they have large data size. So, compression of medical images is needed for effective storage and transmission. We have to protect medical images to prevent making bad uses of them. However, region of interest (RoI) of medical image should not be damaged in processing of compression and protection. Medical image segmentation is needed to compress and protect medical images without the damage of RoI. However, image segmentation is difficult for a radiologist to manually segment the large size of data, and because of the similarity of the biological characteristics of human organs, accurate medical image segmentation is not easy. So, in the field of medical image segmentation, many researchers are studying a variety of ways to obtain fast and accurate automatic segmentation methods for medical images.

Many methods such as threshold method, watershed, region growing, active shape models (ASM), clustering, and level-set method have been researched for medical image segmentation [16-22]. In performing segmentation, accurate judgment is necessary in order to exactly extract the region of interest from medical images in the presence of other organs. For example, if you want to segment the lung region in chest CT images, the bronchi can be a segment that exists within a chest CT image. In case sufficient information is obtained from the region of the lungs and bronchial region, the segmentation can be performed accurately distinguishing the two regions. On the other hand, in case the size of the region, as shown in Figure 2, is small, the region could not be determined as lung region by the lack of information for selecting the lung region.

In this paper, we researched how to improve the performance of exact segmentation of a small region with volume data which is a bunch of medical images. First, we perform initial segmentation. Small regions are damaged or removed in the initial segmentation process. Damaged or removed small regions need reconstruction to improve performance of segmentation. Therefore, we generate predicted segmentation of slices using volume data with linear equation and proposed improvement method for small regions using the predicted segmentation. Using chest CT images among the medical images, we improved the segmentation result and evaluated the performance through the proposed method. 

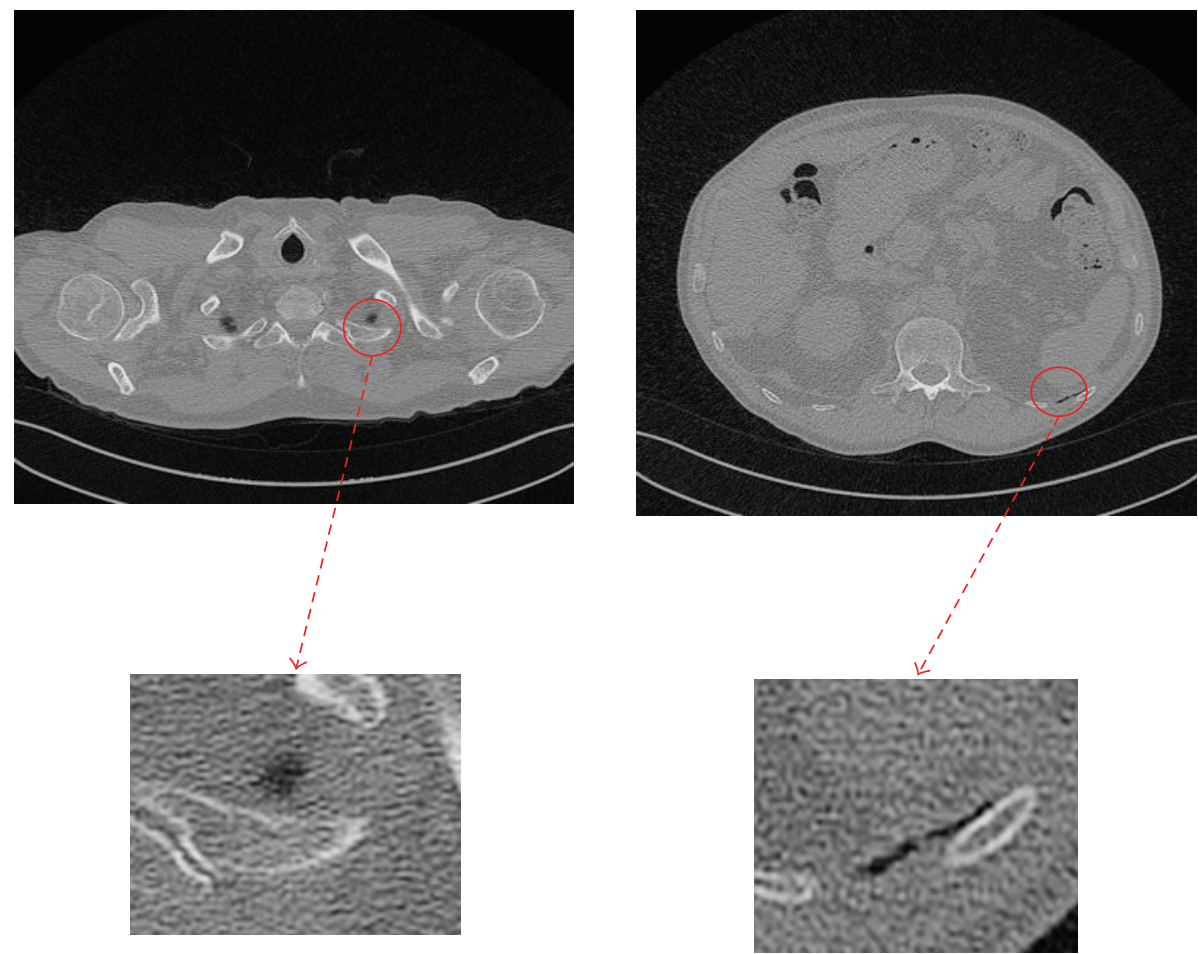

FIGURE 2: The small lung regions.

This paper is organized as follows. In Section 2, proposed improvement method is described. Experiment result is given in Section 3 and conclusion for this paper is made in Section 4 .

\section{Proposed Improvement Method Using Volume Data and Linear Equation}

2.1. Chest CT Image. The screening of the lungs is important since lung cancer death rate is high among cancers. Among the chest imaging methods, radiograph is a common early screening method which has the advantages of low dose and low cost. In radiograph which expresses a chest on a single image, shadow is generated according to anatomical structures such as ribs and heart. Because radiograph consists of a single image, it is difficult to distinguish pulmonary vascular and lung nodules. By contrast, detection sensitivity of lung nodule using CT is higher than radiograph since CT generates images with volume data. Nation Cancer Institute published the result of research which lowers lung cancer death rate using $\mathrm{CT}$ rather than using radiographs in screening [23].

Chest CT images of human body use the 12-bit images instead of general 8-bit images. Generally 300 500 chest $\mathrm{CT}$ images region is obtained from a patient, and it varies depending on the performance of the CT scan equipment. Figure 3 shows the representation of three-dimensional modeling of lungs. Because the top and bottom parts of the lungs have diminishing structure becoming smaller and smaller, the lung region of the top and bottom is small. It is difficult

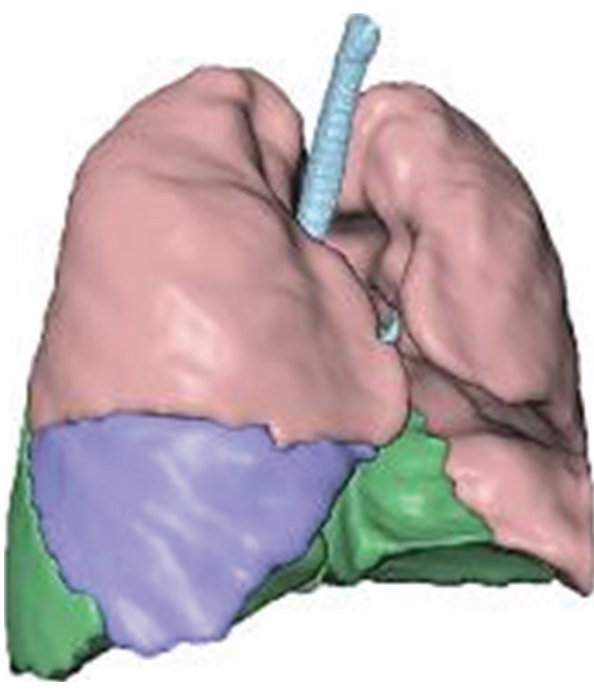

FIGURE 3: Representation of 3D lung modeling.

to determine and segment lung region because the small region of the top and bottom of the lungs does not have many features of the lung.

Chest CT images of the dataset consist of axial chest image slices as shown in Figures 4(a) and 4(b). The bundle of axial chest CT images comprising dataset of chest image is able to generate a volume data. Also, it is able to generate the coronal chest image through the volume data as shown in Figure 4(c). Through coronal chest CT image, we can find that shape of lung image does not consist of dramatic changes but 

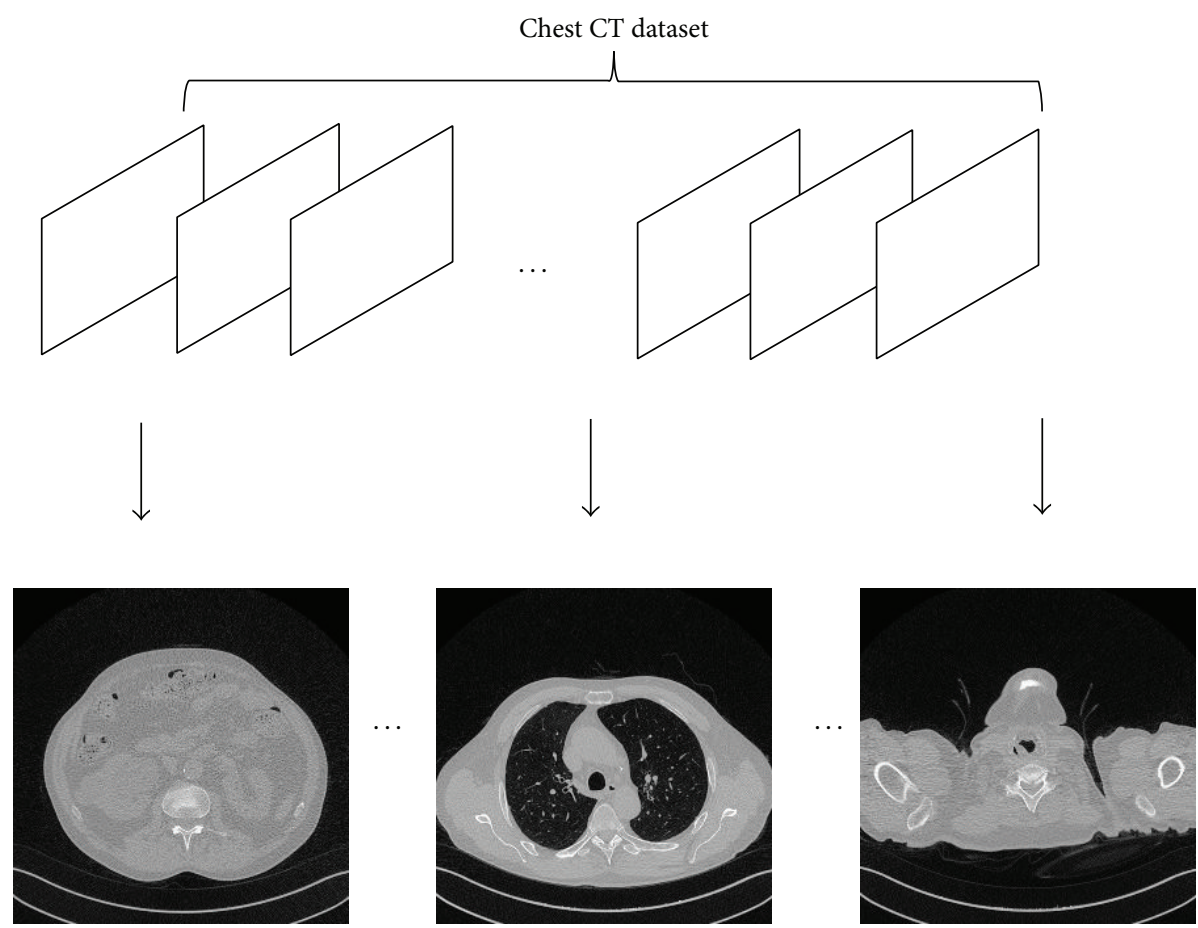

(a) The dataset of chest CT images

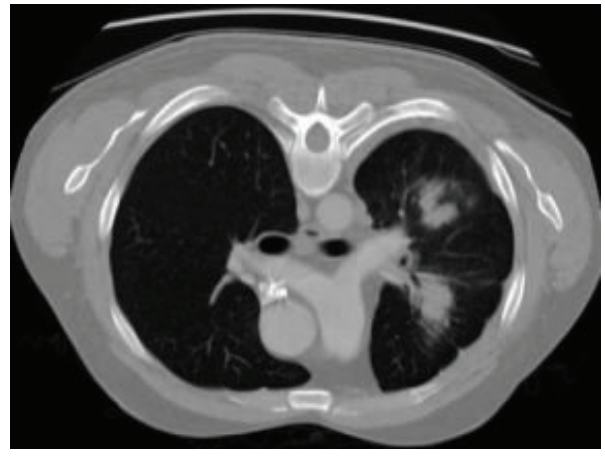

(b) Axial chest CT image

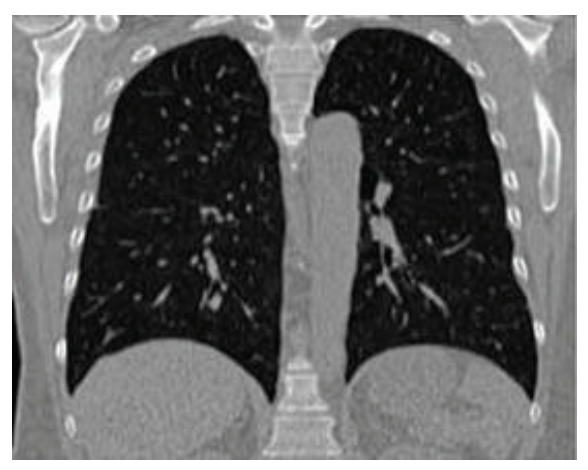

(c) Coronal chest CT image

FIGURE 4: The configuration of chest CT dataset.

naturally connected slices. It means that the previous form of lung slices and the next slices are similar to each other and there are natural connections. Using such chest CT images of volume data, predictions of small lung region can reconstruct segmentation results. Accordingly, the performance of lung segmentation was improved.

2.2. Linear Equation. The connection form of the coronal plane of the lungs does not have a complex shape. And dramatic changes do not occur because a space between slices is narrow. Therefore, in this paper, segmentation region of next slice is predicted using information of reference slice. To predict region change of next slice, we do not use a method that projects reference slice to next slice but use a method that predicts change of region using linear equation. In addition, because the information of the next slice is predicted using reference slices, lung region was predicted without using higher-order linear equations, but by the first linear equation which has less computation. The first equation or linear equation is an equation with the highest order term of the order of 1 . The first equation may have more than one variable. Linear equations with two variables are actually linear functions as shown in Figure 5. In addition, this is called "equation of the straight line" because it is a straight line in the coordinate plane and associated with the geometric properties of the straight lines.

If given two different points $\left(x_{1}, f_{1}\right)$ and $\left(x_{2}, f_{2}\right)$, the equation of a straight line is defined as follows:

$$
\begin{aligned}
& f_{1}=a x_{1}+b, \\
& f_{2}=a x_{2}+b .
\end{aligned}
$$




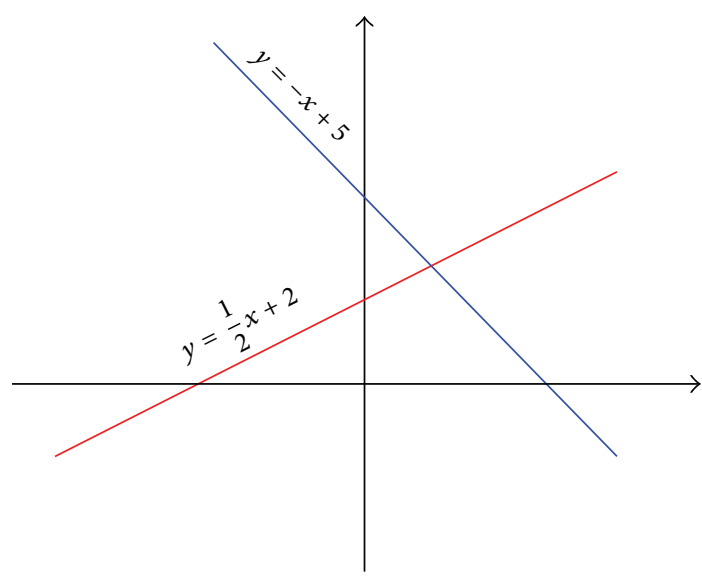

FIgURE 5: The graph of linear equation.

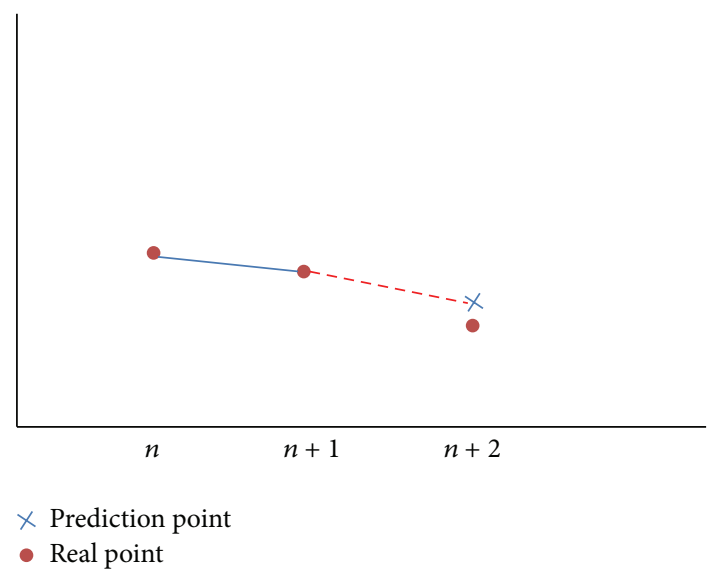

FIgURE 6: Principles of prediction using linear equations.

Using (1), a random coordinate $(x, f)$ on the line can be obtained as the following equation:

$$
\begin{gathered}
a=\frac{f_{2}-f_{1}}{x_{2}-x_{1}}, \\
b=f_{2}-\left(\frac{f_{2}-f_{1}}{x_{2}-x_{1}}\right) x_{2}, \\
f=\left(\frac{f_{2}-f_{1}}{x_{2}-x_{1}}\right)\left(x-x_{1}\right)+f_{1} .
\end{gathered}
$$

That is, given $\left(x_{1}, f_{1}\right)$ and $\left(x_{2}, f_{2}\right),\left(x_{n}, f_{n}\right)$ is able to be predicted through the linear equations. As shown in Figure 6, after contour information of lung is extracted in the $n$th and $n+1$ th slices of chest CT images, the coordinates similar to the actual contours coordinates can be obtained if the outline of the results of the $n+2$ th second installment is predicted through the linear equation.

2.3. Improvement of Segmentation. Figure 7 is a flow chart of the whole, reconstructing the segmentation results to improve performance of segmentation. First, perform initial segmentation of lung region of each slice of chest CT image dataset. In order to set the reference slices, measure the dispersedness of each slice and select two consecutive slices with the lowest dispersedness. After selecting anchor points on the contours of the reference slices, adjust results for the segmentation.

In order to apply linear equations to segment region, the coordinate information of contour should be extracted. Using the coordinate information of all contour points is not efficient. So, after setting the anchor point at regular intervals, linear equations between the anchor points should be obtained. As shown in Figure 8(a), set the anchor points on contour of the initial segmentation results of $n$ and $n+1$ th slices at regular intervals. Then, correlation pairs of anchor points are selected using the distance anchor points between $n$ and $n+1$ th slices. Here, the pair of the shortest distance will become a correlation pair of anchor point. Figure $8(\mathrm{~b})$ shows the difference between the initial (dotted line of red) and predicted (line of blue) segmentation results. After searching for a pair of fixed points of the shortest distance between the two results, use the initial segmentation results if they exist within a certain distance. On the other hand, if the initial segmentation results do not exist within a certain distance, adjust segmentation results using the predicted results as the final result.

Lung region can be generated on lung slices in which the region does not exist using a linear equation to predict the contour of the segmentation results. Lung candidate region information was generated by the threshold in order to solve this problem, and the predictions were applied. Using reference image $m_{n}$ and $m_{n+1}$, generate $P_{n+2}$ segmentation prediction. Here, $m_{n}$ is initial segmentation result of $n$th slice. Then, predicted segmentation $P_{n+2}$ is combined with $m_{n+2}$ to improve segmentation result of $m_{n+2}$. As a result of combining $P_{n+2}$ and $m_{n+2}$, we generate $M_{L} . M_{L}$ is able to be included artificial regions, since $P_{n+2}$ is not real result but predicted result. In order to reduce error such as artificial regions, segmentation information $T_{n+2}$ using threshold was used. $T_{n+2}$ is initial segmentation information which contains all the regions of lung and bronchi. $T_{n+2}$ is combined with $M_{L}$ using AND operation. Therefore, the errors that generate a lung region in slice which has not real lung region do not occur in final segmentation result $I_{n+2}$. This can be expressed as the following equation:

$$
\begin{gathered}
P_{n+2}=F\left(m_{n}, m_{n+1}\right), \\
M_{L}=P_{n+2}+m_{n+2}, \\
I_{n+2}=M_{L} \cap T_{n+2} .
\end{gathered}
$$

In order to obtain such linear equations at least two reference slices are needed. Objects having simpler shapes are the lower probability of segmentation fault. Therefore, we used the dispersedness which can express the simplicity of the form in a numerical value to automatically select the reference slice. By the perimeter of the image $(p)$, and the region of the image $(a)$, the dispersedness can be summarized 


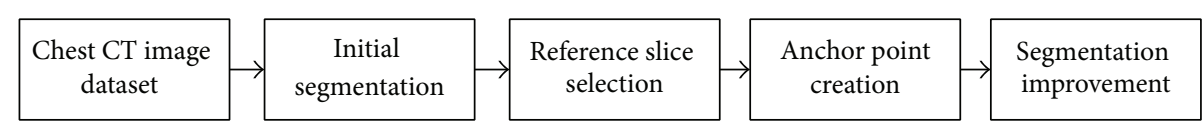

FIgURE 7: The flow chart of proposed segmentation method.

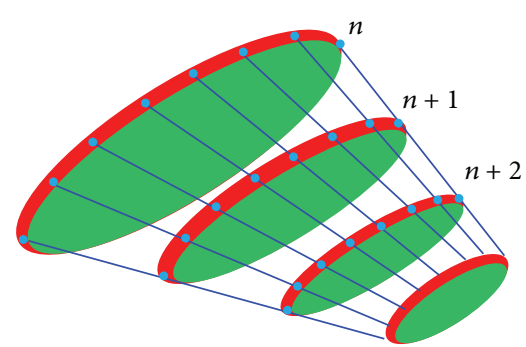

(a) Building a linear equation

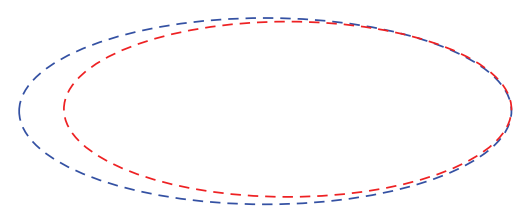

(b) Initial results and the predicted results

FIGURE 8: Calibration process using a linear equation.

as follows [24]. The lower dispersedness means the object with the simpler shape:

$$
D=\frac{p^{2}}{a}
$$

The results of performing segmentation improvement generate rough segmentation results because interval occurs between anchor points set for the linear equation. Therefore, an extension of the segmentation results fit to the object is necessary. In this paper, the segmentation results using the level-set segmentation method were extended to fit the object.

\section{Experimental Results}

In this paper, to improve the performance of segmentation Vessel Segmentation in the Lung, 2012 (VESSEL12) DB using experiments, was carried out. The VESSEL12 was held as a workshop of International Symposium on Biomedical Imaging 2012 (ISBI2012) introduced through the Grand Challenges in Medical Image Analysis [25]. VESSEL12 DB consists of a total of 20 chest CT image dataset and segmentation mask dataset which is lung region segmentation information. Chest CT images in VESSEL12 dataset are composed of $512 \times$ $512 \times 12$ bit images. The segmentation mask file consists of a $512 \times 512 \times 8$ bit and lung region was classified as 0,1 . One dataset consists of an average of 430 slices and a total of 8,593 chest CT image slices. Dice's overlap methods were used to measure lung region segmentation performance. Result of lung segmentation $A$ and segmentation mask image $B$ in
TABLE 1: Experiment result.

\begin{tabular}{lcc}
\hline & $\begin{array}{c}\text { Segmentation without } \\
\text { proposed method }\end{array}$ & $\begin{array}{c}\text { Segmentation with } \\
\text { proposed method }\end{array}$ \\
\hline$S$ & 0.978 & 0.981 \\
Standard & 0.281 & 0.187 \\
Q1 & 0.979 & 0.982 \\
Median & 0.980 & 0.981 \\
\hline
\end{tabular}

VESSEL12 DB was calculated using the following equation [26]:

$$
\text { Score }=\frac{2(A \cap B)}{(A+B)} .
$$

Figure 9 shows the appearance that did not segment small lung region and the result of the proposed method reconstructs the segmentation results. Before using the proposed method, small lung region was removed in the segmentation results as shown in Figure 9(b), in the lung segmentation process and lung region determination process. However, the segmentation improvement method using volume data and linear equations shows segmentation result restoring the small lung region as shown in Figure 9(c).

Level-set method was used as the method to initial segmentation for medical images; DRLS was used for the speed function of the level-set method [27]. Table 1 shows the performance of the segmentation method with and without the proposed method. Score for chest CT imaging of a volume data $(S)$ was measured using Dice's overlap, standard deviation of score (Std), and first quartile (Q1), and median for each slice was measured. Q1 is the median of the lower half of the data set. This means that about $25 \%$ of the numbers in the data set lie below Q1 and about 75\% lie above Q1. Compared to the conventional method, score of the proposed method was improved from 0.978 to 0.981 and the standard deviation was improved from 0.281 to 0.187 . Also, we confirmed to improve performance of segmentation of each slice through reduced Q1 and median. Because the size of the improved segmentation region through proposed improvement method was small, the overall accuracy of the impact was small. But, as shown in Figure 9, even in the slice of which lung region is too small to perform lung region segmentation, lung region segmentation was performed.

\section{Conclusions}

As the performance of medical imaging equipment is improving, medical diagnostic using a computer-assisted image analysis is becoming more important. Telemedicine and IoT enable that specialist can consult the patient's condition despite they are in different place. Also, as the specialist uses 


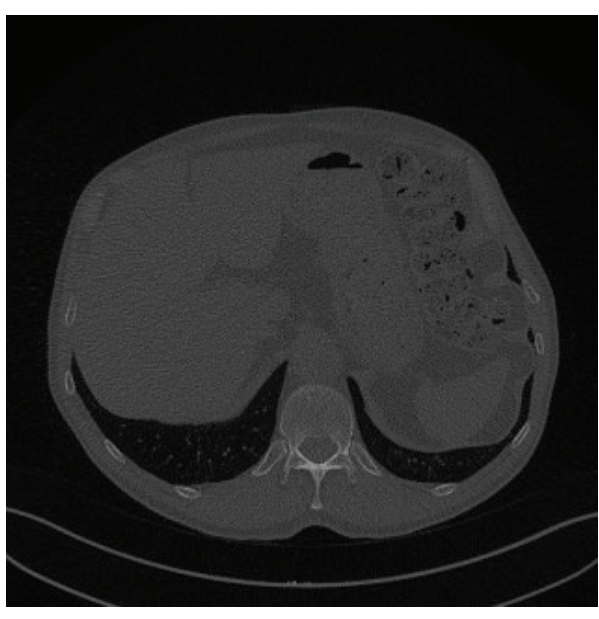

(a) Original CT image

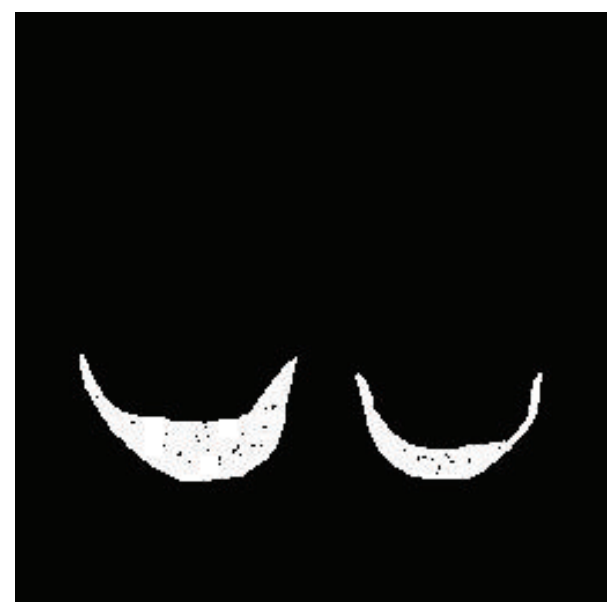

(b) Initial segmentation result

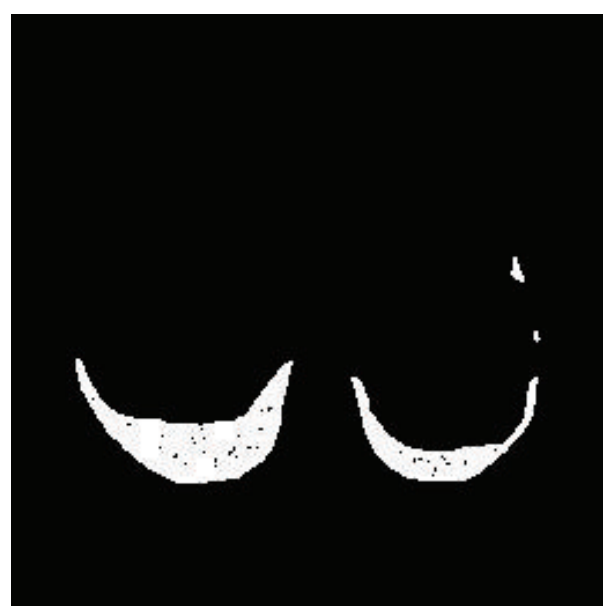

(c) Result of proposed method

FIGURE 9: Result of lung segmentation improvement.

CAD auxiliary, researches published about what can enhance effect of diagnosis and treatment by using CAD accessorily. In order to effectively use the medical images, many researchers have been researching a variety of methods for fast and accurate segmentation of medical images. In performing segmentation, accurate judgment of region is necessary in order to exactly extract the region of interest from medical images in the presence of other organs. However, the damaged or removed regions occur by the lack of information to determine the interest region in a small region. Damaged or removed small regions need reconstruction to improve performance of segmentation in medical images. Because the top and bottom parts of the lungs have diminishing structure becoming smaller and smaller, the lung region of the top and bottom is small. It is difficult to determine and segment lung region because small region of the top and bottom of the lungs does not have many features of the lung.

In this paper, we researched how to reconstruct the performance of exact segmentation of small region with volume data and linear equation. The performance of segmentation can be improved through reconstruction of small lung region. Through coronal lung image, we can find that shape of lung image does not consist of dramatic changes but naturally connected slices. Therefore, linear equations using two reference slices can predict the segmentation region of the next slice. Using dispersedness of initial segmentation results, two reference slices were selected, and then anchor points were set on the contour of initial segmentation region in the slices. After obtaining a linear equation using a pair of anchor points in the two slices, segmentation region of the next slice of the reference slice was predicted. By the combination of the predicted results and the initial segmentation result, segmentation of small region was reconstructed. As a result of experiment, we could confirm restructuring damaged or removed small lung regions in chest CT images. And performance of segmentation was improved from 0.978 to 0.981 . In particular, the standard deviation of the slices of the volume data is improved $18.7 \%$ from 0.281 to 0.187 , and 
even improvement of segmentation performance in each slice was confirmed.

In the future, we plan to perform image segmentation using a variety of medical imaging $\mathrm{DB}$ and conduct researches to detect lesions which exist within the segmented region.

\section{Conflict of Interests}

The authors declare that there is no conflict of interests regarding the publication of this paper.

\section{Acknowledgment}

This research was supported by Basic Science Research Program through the National Research Foundation of Korea (NRF) funded by the Ministry of Education, Science and Technology (2011-0023147).

\section{References}

[1] WHO Website, http://www.who.int.

[2] D. Hailey, R. Roine, and A. Ohinmaa, "Systematic review of evidence for the benefits of telemedicine," Journal of Telemedicine and Telecare, vol. 8, no. 1, pp. 1-7, 2002.

[3] P. T. Moore and M. Sharma, "Enhanced patient management in a hospital setting," Journal of IT Convergence Practice, vol. 1, no. 4, pp. 1-23, 2013.

[4] S. Park, D. K. Shin, and J. S. Kim, "Components of computeraided diagnosis for breast ultrasound," Journal of IT Convergence Practice, vol. 1, no. 4, pp. 50-63, 2013.

[5] S. Stowe and S. Harding, "Telecare, telehealth and telemedicine," European Geriatric Medicine, vol. 1, no. 3, pp. 193-197, 2010.

[6] M. Brahami, B. Atmani, and N. Matta, "Dynamic knowledge mapping guided by data mining: application on healthcare," Journal of Information Processing Systems, vol. 9, no. 1, pp. 1-30, 2013.

[7] H. Kim, Y. Kim, and J. Chang, "A grid-based cloaking area creation scheme for continuous LBS queries in distributed systems," Journal of Convergence, vol. 4, article 1, 2013.

[8] A. Shinha and D. K. Lobiyal, "Performance evaluation of data aggregation for cluster-based wireless sensor network," HumanCentric Computing and Information Sciences, vol. 3, article 13, 2013.

[9] X. M. Zhang and C. Xu, "A multimedia telemedicine system in internet of things," in Proceedings of the Computer Science \& Information Technology, vol. 42, pp. 180-187, 2012.

[10] L. Atzori, A. Iera, and G. Morabito, "The internet of things: a survey," Computer Networks, vol. 54, no. 15, pp. 2787-2805, 2010.

[11] W. Shuicai, J. Peijie, Y. Chunlan, L. Haomin, and B. Yanping, "The development of a tele-monitoring system for physiological parameters based on the B/Smodel," Computers in Biology and Medicine, vol. 40, no. 11-12, pp. 883-888, 2010.

[12] D.-Y. Fei, X. Zhao, C. Boanca et al., "A biomedical sensor system for real-time monitoring of astronauts' physiological parameters during extra-vehicular activities," Computers in Biology and Medicine, vol. 40, no. 7, pp. 635-642, 2010.

[13] J. T. Dobbins III, "Tomosynthesis imaging: at a translational crossroads," Medical Physics, vol. 36, no. 6, pp. 1956-1967, 2009.
[14] L. Costaridou, Medical Image Analysis Methods, Taylor \& Francis, 2005.

[15] F. Ritter, T. Boskamp, A. Homeyer et al., "Medical image analysis," IEEE Pulse, vol. 2, no. 6, pp. 60-70, 2011.

[16] Y. Zhu, X. Papademetris, A. J. Sinusas, and J. S. Duncan, "Segmentation of the left ventricle from cardiac MR images using a subject-specific dynamical model," IEEE Transactions on Medical Imaging, vol. 29, no. 3, pp. 669-687, 2010.

[17] H. Badakhshannoory and P. Saeedi, "A model-based validation scheme for organ segmentation in CT scan volumes," IEEE Transactions on Biomedical Engineering, vol. 58, no. 9, pp. 26812693, 2011.

[18] Z. Liu, H. Wang, and Q. Li, “Tongue tumor detection in medical hyperspectral images," Sensors, vol. 12, no. 1, pp. 162-174, 2012.

[19] J. Zhang, C.-H. Yan, C.-K. Chui, and S.-H. Ong, "Fast segmentation of bone in CT images using 3D adaptive thresholding," Computers in Biology and Medicine, vol. 40, no. 2, pp. 231-236, 2010.

[20] M. C. J. Christ and R. M. S. Parvathi, "Segmentation of medical image using K-Means clustering and marker controlled watershed algorithm," European Journal of Scientific Research, vol. 71, no. 2, pp. 190-194, 2012.

[21] P. Kaur, A. K. Soni, and A. Gosain, "A robust kernelized intuitionistic fuzzy c-means clustering algorithm in segmentation of noisy medical images," Pattern Recognition Letters, vol. 34, no. 2, pp. 163-175, 2013.

[22] S. Chen, T. Kohlberger, and K. J. Kirchberg, "Advanced level set segmentation of the right atrium in MR," in Medical Imaging: Visualization, Image-Guided Procedures, and Modeling, vol. 7964 of Proceedings of SPIE, February 2011.

[23] P. B. Bach, J. N. Mirkin, T. K. Oliver et al., "Benefits and harms of CT screening for lung cancer: a systematic review," The Journal of the American Medical Association, vol. 307, no. 22, pp. 24182429, 2012.

[24] A. J. Liption, "Moving target classification and tracking from real-time video," in Proceedings of the Applications of Computer Vision, pp. 8-14, 1998.

[25] VESsel Segmentation the Lung 2012 (VESSEL12), http:// vessel12.grand-challenge.org.

[26] L. Dice, "Measures of the amount of ecologic association between species," Ecology, vol. 26, no. 3, pp. 297-302, 1945.

[27] C. Li, C. Xu, C. Gui, and M. D. Fox, "Distance regularized level set evolution and its application to image segmentation," IEEE Transactions on Image Processing, vol. 19, no. 12, pp. 3243-3254, 2010. 


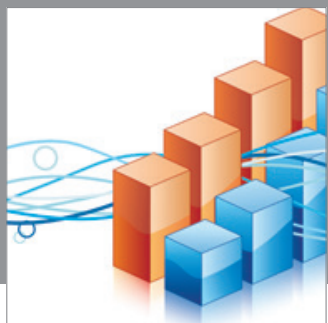

Advances in

Operations Research

mansans

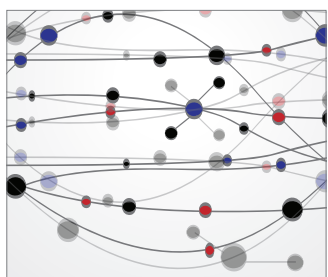

The Scientific World Journal
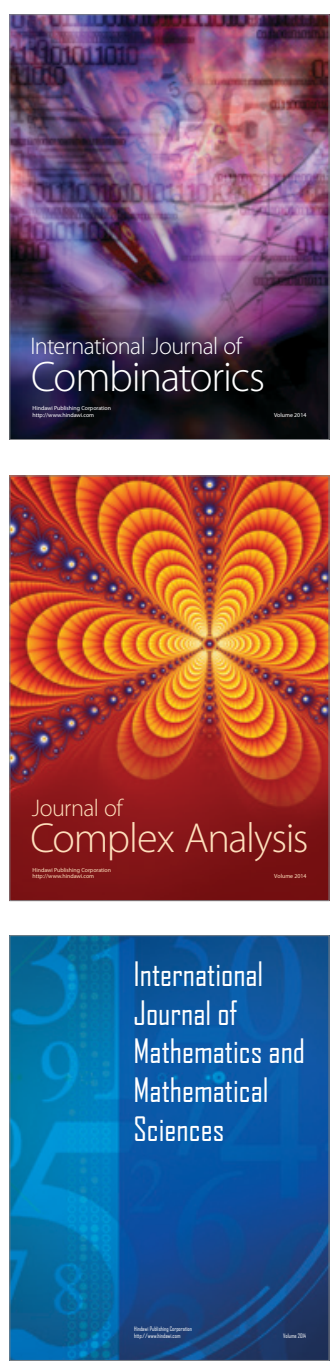
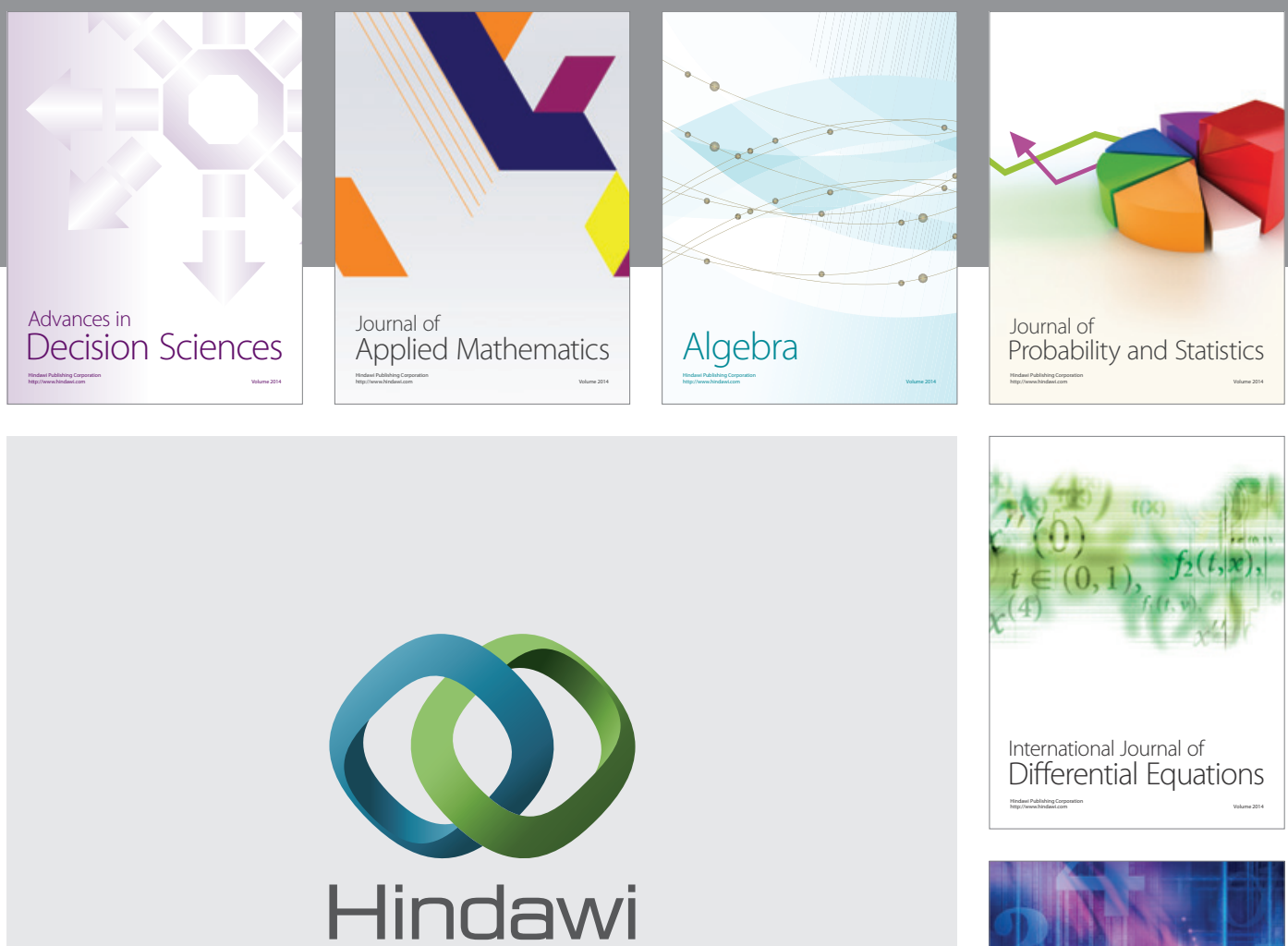

Submit your manuscripts at http://www.hindawi.com
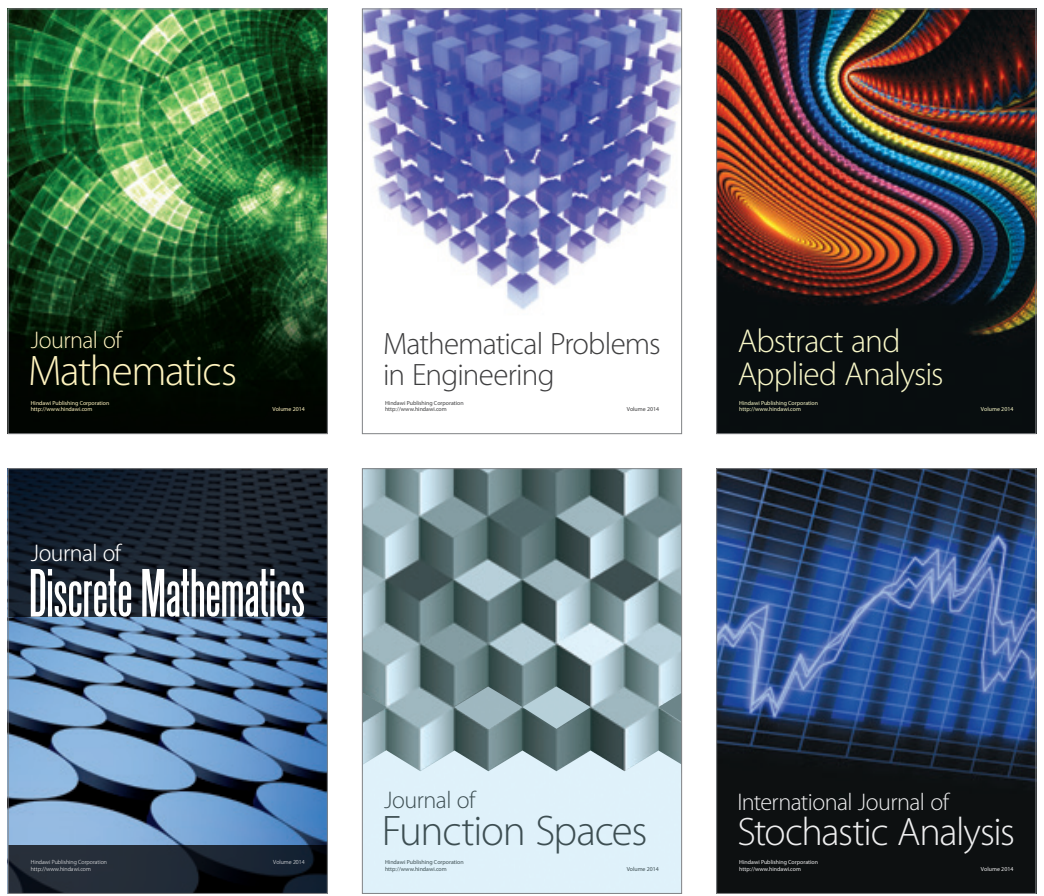

Journal of

Function Spaces

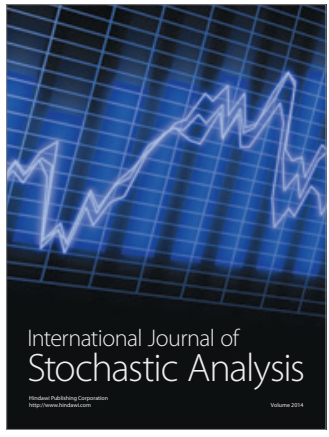

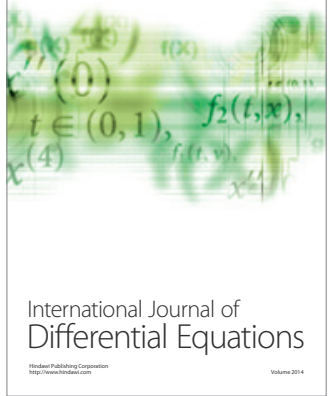
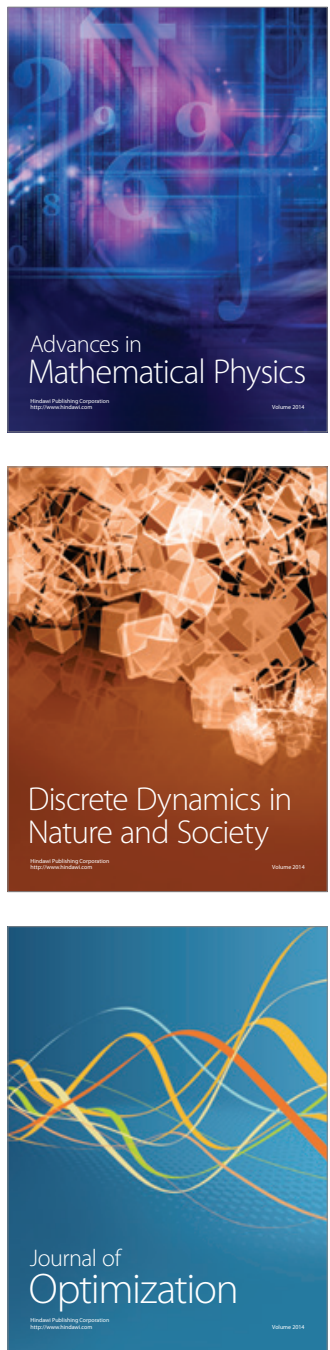